\title{
Sard's approximation processes and oblique projections
}

by

\author{
G. Corach, J. I. Giribet, and A. Maestripieri (Buenos Aires)
}

\begin{abstract}
Three problems arising in approximation theory are studied. These problems have already been studied by Arthur Sard. The main goal of this paper is to use geometrical compatibility theory to extend Sard's results and get characterizations of the sets of solutions.
\end{abstract}

1. Introduction. In 1950, Arthur Sard [17] proposed an operator-theoretic approach to study some problems arising in approximation theory. In his terminology, a process is an operator $T$ on a Hilbert space $\mathcal{H}$ which is used to approximate $x \in \mathcal{H}$ in the sense that if $\delta x$ is the error, then $T(x+\delta x)$ approximates $x$. For a fixed closed subspace $\mathcal{S}$ of $\mathcal{H}$, he studied approximation processes over $\mathcal{S}$, least squares processes on $\mathcal{S}$ and curve fitting processes on $\mathcal{S}$ (see definitions below).

Recall that a positive semidefinite operator $A$ and a closed subspace $\mathcal{S}$ are called compatible if there exists a (bounded linear) projection $Q$ with range $\mathcal{S}$ which is self-adjoint with respect to the sesquilinear form $\langle\xi, \eta\rangle_{A}=$ $\langle A \xi, \eta\rangle$ for every $\xi, \eta \in \mathcal{H}$, i.e., $A Q=Q^{*} A$. Denote by $\mathcal{P}(A, \mathcal{S})$ the set of all projections $Q$ such that $A Q=Q^{*} A$ and $R(Q)=\mathcal{S}$. If $\mathcal{P}(A, \mathcal{S})$ is not empty, it contains a distinguished element, denoted $P_{A, \mathcal{S}}$, which has nullspace $A(\mathcal{S})^{\perp} \ominus(\mathcal{S} \cap N(A))$ (here $\left.\mathcal{M} \ominus \mathcal{N}=\mathcal{M} \cap(\mathcal{M} \cap \mathcal{N})^{\perp}\right)$. Analogously, denote by $\mathcal{P}_{*}(A, \mathcal{S})$ the set of all projections such that $A Q^{*}=Q A$ and $R(Q)=\mathcal{S}$. The notion of compatibility is related to Schur complements $[1,8,9,14]$, Ando complementability [2, 16], abstract splines in Hilbert spaces $[6,10,11,15]$, weighted pseudo inverses [7], frame theory [5], signal processing $[12,13]$, sampling theory $[4,18]$, and so on.

It turns out that compatibility allows a geometrical approach to the technical results of Sard. The main goal of this paper is to characterize the sets of processes defined by Sard in terms of certain compatibility conditions.

2000 Mathematics Subject Classification: Primary 47A58, 41A65; Secondary 93E24.

Key words and phrases: oblique projections, weighted least square problems, approximation processes. 
The main results of the paper are the following:

(a) The set of $A$-approximation processes over $\mathcal{S}$ is not empty if and only if $A$ is compatible with $\mathcal{S}^{\perp}$; moreover, it coincides with $\mathcal{P}_{*}\left(A, \mathcal{S}^{\perp}\right)+$ $L(N(A) \cap \mathcal{S}, \mathcal{S})$.

(b) There exists an $A$-weighted least squares process on $\mathcal{S}$ if and only if $A$ and $\mathcal{S}$ are compatible; in this case, these processes are operators of the form $\mathcal{P}_{A, \mathcal{S}}+T$ with $T \in L(\mathcal{H}, \mathcal{S} \cap N(A))$.

(c) Given positive operators $A, B \in L(\mathcal{H})^{+}$and a closed subspace $\mathcal{S}$ of $\mathcal{H}$ there exists a $B$-approximation process which is also an $A$-weighted least squares process on $\mathcal{S}$ if and only if $(A, \mathcal{S})$ is compatible and the Dixmier angle between $N(A) \cap \mathcal{S}$ and $B\left(\mathcal{S}^{\perp}\right)$ is positive.

(d) Given a positive trace class operator $A \in L(\mathcal{H})$ and a closed subspace $\mathcal{S}$ of $\mathcal{H}$, there exists an $A$-curve fitting process on $\mathcal{S}$ if and only if $\left(A, \mathcal{S}^{\perp}\right)$ is compatible. Furthermore, we give a characterization of these processes.

2. Preliminaries. Along this work, $\mathcal{H}$ denotes a (complex, separable) Hilbert space with inner product $\langle$,$\rangle . Given two Hilbert spaces \mathcal{H}$ and $\mathcal{K}$, $L(\mathcal{H}, \mathcal{K})$ is the space of bounded linear operators from $\mathcal{H}$ into $\mathcal{K}$, and $L(\mathcal{H})=$ $L(\mathcal{H}, \mathcal{H})$. If $T \in L(\mathcal{H}, \mathcal{K})$ then $T^{*} \in L(\mathcal{K}, \mathcal{H})$ denotes the adjoint operator of $T, R(T)$ stands for the range of $T$, and $N(T)$ for its nullspace. If $\mathcal{S}$ is a closed subspace of $\mathcal{H}$ and $\mathcal{T}$ is a closed subspace of $\mathcal{K}$, then $L(\mathcal{S}, \mathcal{T})$ will be identified with the subspace of $L(\mathcal{H}, \mathcal{K})$ consisting of all $T \in L(\mathcal{H}, \mathcal{K})$ such that $R(T) \subseteq \mathcal{T}$ and $\mathcal{S}^{\perp} \subseteq N(T)$.

Let $L(\mathcal{H})^{+}$be the cone of (semidefinite) positive operators of $L(\mathcal{H})$ and denote by $\mathcal{Q}$ the set of projections of $L(\mathcal{H})$, i.e., $\mathcal{Q}=\left\{Q \in L(\mathcal{H}): Q^{2}=Q\right\}$.

If $\mathcal{S}$ and $\mathcal{T}$ are two (closed) subspaces of $\mathcal{H}$, denote by $\mathcal{S}+\mathcal{T}$ the direct sum of $\mathcal{S}$ and $\mathcal{T}$, by $\mathcal{S} \oplus \mathcal{T}$ their (direct) orthogonal sum, and set $\mathcal{S} \ominus \mathcal{T}=$ $\mathcal{S} \cap(\mathcal{S} \cap \mathcal{T})^{\perp}$. If $\mathcal{H}=\mathcal{S} \dot{+} \mathcal{T}$, the oblique projection $P_{\mathcal{S} / / \mathcal{T}}$ onto $\mathcal{S}$ along $\mathcal{T}$ is the projection with $R\left(P_{\mathcal{S} / / \mathcal{T}}\right)=\mathcal{S}$ and $N\left(P_{\mathcal{S} / / \mathcal{T}}\right)=\mathcal{T}$. In particular, $P_{\mathcal{S}}=P_{\mathcal{S} / / \mathcal{S}^{\perp}}$ is the orthogonal projection onto $\mathcal{S}$.

Given two subspaces $\mathcal{S}, \mathcal{T}$, the cosine of the Friedrichs angle $\theta(\mathcal{S}, \mathcal{T}) \in$ $[0, \pi / 2]$ between them is defined by

$$
c(\mathcal{S}, \mathcal{T})=\sup \{|\langle x, y\rangle|: x \in \mathcal{S} \ominus \mathcal{T},\|x\|<1, y \in \mathcal{T} \ominus \mathcal{S},\|y\|<1\} .
$$

The following conditions are equivalent:

(1) $c(\mathcal{S}, \mathcal{T})<1$

(2) $\mathcal{S}+\mathcal{T}$ is closed;

(3) $\mathcal{S}^{\perp}+\mathcal{T}^{\perp}$ is closed;

(4) $c\left(\mathcal{S}^{\perp}, \mathcal{T}^{\perp}\right)<1$. 
The Dixmier angle between $\mathcal{S}$ and $\mathcal{T}$ is the angle in [0, $\pi / 2]$ whose cosine is defined by

$$
c_{0}(\mathcal{S}, \mathcal{T})=\sup \{|\langle x, y\rangle|: x \in \mathcal{S},\|x\|<1, y \in \mathcal{T},\|y\|<1\} .
$$

Observe that in general $c(\mathcal{S}, \mathcal{T}) \leq c_{0}(\mathcal{S}, \mathcal{T})$, and if $\mathcal{S} \cap \mathcal{T}=\{0\}$ then equality holds. Notice that if $c_{0}(\mathcal{S}, \mathcal{T})<1$ then $\mathcal{S} \cap \mathcal{T}=\{0\}$.

Given $A \in L(\mathcal{H})^{+}$consider the (bounded) sesquilinear form in $\mathcal{H} \times \mathcal{H}$ defined by

$$
\langle x, y\rangle_{A}=\langle A x, y\rangle \quad \text { for } x, y \in \mathcal{H},
$$

and the corresponding seminorm $\|x\|_{A}^{2}=\langle x, x\rangle_{A}$.

If $\mathcal{S}$ is a closed subspace of $\mathcal{H}$ and $A \in L(\mathcal{H})^{+}$, the $A$-orthogonal subspace to $\mathcal{S}$ is given by

$$
\mathcal{S}^{\perp_{A}}:=\left\{x \in \mathcal{H}:\langle x, s\rangle_{A}=0 \text { for every } s \in \mathcal{S}\right\} .
$$

We have $\mathcal{S}^{\perp_{A}}=A^{-1}\left(\mathcal{S}^{\perp}\right)=A(\mathcal{S})^{\perp}$.

An operator $T \in L(\mathcal{H})$ is $A$-selfadjoint if $\langle T x, y\rangle_{A}=\langle x, T y\rangle_{A}$ for every $x, y \in \mathcal{H}$. It is easy to see that $T$ satisfies this condition if and only if $A T=T^{*} A$.

Definition 2.1. Let $A \in L(\mathcal{H})^{+}$and $\mathcal{S}$ be a closed subspace of $\mathcal{H}$. The pair $(A, \mathcal{S})$ is compatible if there exists an $A$-selfadjoint projection with range $\mathcal{S}$, i.e., the set

$$
\mathcal{P}(A, \mathcal{S})=\left\{Q \in \mathcal{Q}: R(Q)=\mathcal{S}, A Q=Q^{*} A\right\}
$$

is not empty.

Observe that a projection $Q$ is $A$-selfadjoint if and only if $N(Q) \subseteq$ $R(Q)^{\perp_{A}}$. It easily follows that $(A, \mathcal{S})$ is compatible if and only if

$$
\mathcal{H}=\mathcal{S}+A^{-1}\left(\mathcal{S}^{\perp}\right)
$$

Given a compatible pair $(A, \mathcal{S})$, let $\mathcal{N}=\mathcal{S} \cap A(\mathcal{S})^{\perp}$. It is easy to see that $\mathcal{N}=\mathcal{S} \cap N(A)$. The decomposition $\mathcal{H}=\mathcal{S} \dot{+}\left(A(\mathcal{S})^{\perp} \ominus \mathcal{N}\right)$ defines the oblique projection

$$
P_{A, \mathcal{S}}=P_{\mathcal{S} / / A(\mathcal{S})^{\perp} \ominus \mathcal{N}} .
$$

Since $R\left(P_{A, \mathcal{S}}\right)=\mathcal{S}$ and $N\left(P_{A, \mathcal{S}}\right) \subseteq A(\mathcal{S})^{\perp}$ it follows that $P_{A, \mathcal{S}} \in \mathcal{P}(A, \mathcal{S})$.

The set $\mathcal{P}(A, \mathcal{S})$ is an affine manifold that, for a given $P \in \mathcal{P}(A, \mathcal{S})$, can be parametrized as

$$
\mathcal{P}(A, \mathcal{S})=P+L\left(\mathcal{S}^{\perp}, \mathcal{N}\right) .
$$

For proofs of these facts see $[8,9]$.

The following list contains examples of compatible and noncompatible pairs. 
Example 2.2. Suppose that $A \in L(\mathcal{H})^{+}$and $\mathcal{S}$ is a closed subspace of $\mathcal{H}$,

(a) If $A$ has closed range, the pair $(A, \mathcal{S})$ is compatible if and only if $N(A)+\mathcal{S}$ is closed, or equivalently $c(N(A), \mathcal{S})<1$.

(b) If $R\left(P_{\mathcal{S}} A P_{\mathcal{S}}\right)$ is closed then the pair $(A, \mathcal{S})$ is compatible.

(c) In particular, if $P_{\mathcal{S}} A P_{\mathcal{S}}$ is invertible in $L(\mathcal{S}, \mathcal{S})$ then the pair $(A, \mathcal{S})$ is compatible, and $\mathcal{P}(A, \mathcal{S})=\left\{P_{A, \mathcal{S}}\right\}$. See [3] for a proof.

(d) If $\mathcal{S}$ has finite dimension then $(A, \mathcal{S})$ is compatible. This follows directly from (a).

(e) $c_{0}\left(\mathcal{S}^{\perp}, \overline{A(\mathcal{S})}\right)<1$ if and only if the pair $(A, \mathcal{S})$ is compatible.

An interesting example where the pair $(A, \mathcal{S})$ is not compatible can be found in [17, Example 12].

The next two concepts were introduced by A. Sard in [17] in order to establish necessary and sufficient conditions for the existence of operators such as approximation, least squares and curve fitting processes.

Definition 2.3. Let $\mathcal{T}$ be a closed subspace of $\mathcal{H}$. An operator $C \in$ $L(\mathcal{H})$ is proper on $\mathcal{T}$ if there exists $D \in L(\mathcal{H})$ such that $D P_{\mathcal{T}} C P_{\mathcal{T}}=P_{\mathcal{T}}$.

Definition 2.4. Let $A \in L(\mathcal{H})^{+}$and $\mathcal{T}$ be a closed subspace of $\mathcal{H}$. An operator $C \in L(\mathcal{H})$ is a companion of $A$ relative to $\mathcal{T}$ if it is proper on $\mathcal{T}$ and $R\left(A C^{*} P_{\mathcal{T}}\right) \subseteq P_{\mathcal{T}}$.

There is a close relationship between the compatibility of the pair $(A, \mathcal{S})$ and the existence of companions of $A$ relative to $\mathcal{S}^{\perp}$; in fact, these two concepts are equivalent.

Proposition 2.5. Let $A \in L(\mathcal{H})^{+}$and $\mathcal{S}$ be a closed subspace of $\mathcal{H}$. There exists a companion of $A$ relative to $\mathcal{S}^{\perp}$ if and only if the pair $(A, \mathcal{S})$ is compatible.

Proof. Suppose that the pair $(A, \mathcal{S})$ is compatible and let $E=I-Q^{*}$ for $Q \in \mathcal{P}(A, \mathcal{S})$; obviously $E$ is a projection and $R(E)=N\left(Q^{*}\right)=R(Q)^{\perp}=\mathcal{S}^{\perp}$. Then $E P_{\mathcal{S}^{\perp}} E P_{\mathcal{S}^{\perp}}=P_{\mathcal{S}^{\perp}}$, i.e., $E$ is proper on $\mathcal{S}^{\perp}$. Furthermore,

$$
A E^{*}=A(I-Q)=A-Q^{*} A=E A,
$$

hence $R\left(A E^{*}\right) \subseteq \mathcal{S}^{\perp}$, which implies that $E$ is a companion of $A$ relative to $\mathcal{S}^{\perp}$.

Conversely, suppose that $C \in L(\mathcal{H})$ is a companion of $A$ relative to $\mathcal{S}^{\perp}$; a fortiori, $C$ is proper on $\mathcal{S}^{\perp}$. Let $D \in L(\mathcal{H})$ be such that $D P_{\mathcal{S}^{\perp}} C P_{\mathcal{S}^{\perp}}=P_{\mathcal{S}^{\perp}}$ and consider $Q=\left(I-P_{\mathcal{S}} \perp P_{\mathcal{S}}{ } C\right)^{*}$. It is easy to see that $E=P_{\mathcal{S} \perp} D P_{\mathcal{S}}{ }^{\perp} C$ is a projection with range $\mathcal{S}^{\perp}$. Then $Q$ is a projection with $R(Q)=\mathcal{S}$. Furthermore, since $R\left(A C^{*} P_{\mathcal{S}^{\perp}}\right) \subseteq \mathcal{S}^{\perp}$, we have $E A E^{*}=A E^{*}$, and since $E A E^{*}$ is selfadjoint, it follows that $A E^{*}=E A$, which implies that $A Q=$ $Q^{*} A$, i.e., $Q \in \mathcal{P}(A, \mathcal{S})$. 
In particular, the above proof shows that if $Q$ is an $A$-selfadjoint projection with range $\mathcal{S}^{\perp}$ then $E=I-Q^{*}$ is a companion of $A$ relative to $\mathcal{S}$. However, not every companion is necessarily a projection (see Theorem 3.4 below).

There also exists a link between the compatibility of $(A, \mathcal{S})$ and the condition of $A$ being proper on $\mathcal{S}$, as shown by the following results.

Lemma 2.6. Let $A \in L(\mathcal{H})^{+}$and $\mathcal{S}$ be a closed subspace of $\mathcal{H}$. Then $A$ is proper on $\mathcal{S}$ if and only if $R\left(P_{\mathcal{S}} A P_{\mathcal{S}}\right)=\mathcal{S}$.

Proof. If $A$ is proper on $\mathcal{S}$, then there exists $D \in L(\mathcal{H})$ such that $D P_{\mathcal{S}} A P_{\mathcal{S}}=P_{\mathcal{S}}$. Let $B=P_{\mathcal{S}} A P_{\mathcal{S}}$; then $B \in L(\mathcal{H})^{+}$and $R(B) \subseteq \mathcal{S}$. From $B D^{*}=P_{\mathcal{S}}$, it follows that $R(B)=\mathcal{S}$.

Conversely, from elementary properties of Moore-Penrose inverse, $B^{\dagger} B=P_{N(B)^{\perp}}=P_{\overline{R\left(B^{*}\right)}}$ and $B B^{\dagger}=P_{R(B)}$. Since $B$ is selfadjoint and $R(B)=\mathcal{S}$, it follows that $B B^{\dagger}=B^{\dagger} B=P_{\mathcal{S}}$.

Proposition 2.7. Let $A \in L(\mathcal{H})^{+}$and $\mathcal{S}$ be a closed subspace of $\mathcal{H}$. Then $A$ is proper on $\mathcal{S}$ if and only if $\mathcal{N}=\{0\}$ and $R\left(P_{\mathcal{S}} A P_{\mathcal{S}}\right)$ is closed. In this case $(A, \mathcal{S})$ is compatible, $\mathcal{P}(A, \mathcal{S})=\left\{P_{A, \mathcal{S}}\right\}$ and $P_{A, \mathcal{S}}=P_{\mathcal{S}}(I+$ $\left.D P_{\mathcal{S}} A P_{\mathcal{S}^{\perp}}\right)$, where $D \in L(\mathcal{H})$ satisfies $D P_{\mathcal{S}} A P_{\mathcal{S}}=P_{\mathcal{S}}$.

Proof. Let $B=P_{\mathcal{S}} A P_{\mathcal{S}}$; notice that $N(B)=\mathcal{N} \oplus \mathcal{S}^{\perp}$. From Lemma 2.6, if $A$ is proper on $\mathcal{S}$ then $R(B)=\mathcal{S}$, therefore it is closed. Also, $N(B)=\mathcal{S}^{\perp}$, so that $\mathcal{N}=\{0\}$.

Conversely, if $R(B)$ is closed and $\mathcal{N}=\{0\}$, then $N(B)=\mathcal{S}^{\perp}$ and $\overline{R(B)}=R(B)=\mathcal{S}$. In this case, from [8, Remark 2.12(2)], it follows that $(A, \mathcal{S})$ is compatible. Observe that if $D B=P_{\mathcal{S}}$ then $P_{\mathcal{S}} D P_{\mathcal{S}} B=$ $P_{\mathcal{S}}$ and $R\left(P_{\mathcal{S}} D P_{\mathcal{S}}\right)=\mathcal{S}$. Let $C=P_{\mathcal{S}} D P_{\mathcal{S}}$. Then $B C^{*}=P_{\mathcal{S}}$ so that $P_{\mathcal{S}} C^{*}=B^{\dagger} P_{\mathcal{S}}=B^{\dagger}$, or $C^{*}=B^{\dagger}=C$. From [8, Remark 2.12(1)], we get $P_{A, \mathcal{S}}=P_{\mathcal{S}}+B^{\dagger} P_{\mathcal{S}} A P_{\mathcal{S}^{\perp}}=P_{\mathcal{S}}+P_{\mathcal{S}} D P_{\mathcal{S}} A P_{\mathcal{S}^{\perp}}$.

Notice that if $A$ has closed range, then $A$ is proper on $\mathcal{S}$ if and only if the pair $(A, \mathcal{S})$ is compatible and $\mathcal{N}=\{0\}$, because the compression $P_{\mathcal{S}} A P_{\mathcal{S}}$ has closed range (see [8, Theorem 6.2]).

3. Approximation processes. Let $\mu$ be a Lebesgue-Stieltjes measure on $\mathbb{R}$ and let $\mathcal{H}$ be the Hilbert space $L^{2}(\mu)$. Suppose that $(\Omega, \mathcal{F}, P)$ is a probability space; if $z: \Omega \rightarrow \mathbb{R}$ is $P$-measurable then the expectation of $z$ is $E(z)=\int_{\Omega} z(\omega) d P(\omega)$.

Let $\delta x: \mathbb{R} \times \Omega \rightarrow \mathbb{R}$ be a $\mu \times P$-measurable function such that:

(1) for almost every $t \in \mathbb{R}, E(\delta x(t, \cdot))=0$,

(2) for almost every $\omega \in \Omega, \delta x(\cdot, \omega) \in \mathcal{H}$,

(3) $E\left(\|\delta x\|^{2}\right)=\int_{\Omega} \int_{\mathbb{R}}|\delta x(\omega, t)|^{2} d \mu(t) d P(\omega)<\infty$. 
The variance operator $A \in L(\mathcal{H})^{+}$of $\delta x$ is defined by

$$
A x=E(\langle x, \delta x\rangle \delta x)=\int_{\Omega} \delta x(\omega, \cdot) \int_{\mathbb{R}} \delta x(\omega, t) x(t) d \mu(t) d P(\omega)
$$

for every $x \in \mathcal{H}$. As shown in [17, Lemma 2], the variance operator $A$ is a trace class operator.

In signal processing applications, $x$ is a (finite energy) signal which has to be estimated and $\delta x$ a noise measurement. Given the measurement $x+\delta x$, we have to recover the signal $x$ by means of a filter (i.e., an operator) $T \in L(\mathcal{H})$. In general, the reconstructed signal $T(x+\delta x)$ does not coincide with the signal $x$. It may happen that, at least in a suitable set of signals, the expected value of the reconstructed signal $E(T(x+\delta x))$ coincides with $x$. If there exist many operators that satisfy our requirement, we add a restriction, for instance that the incidence of the noise in the reconstructed signal be minimized. This problem has been studied in [17] and motivates the definition of approximation processes.

Definition 3.1. Given a closed subspace $\mathcal{T}$ of $\mathcal{H}$ and $\delta x$ (with the above assumptions), let $\mathcal{U}=\{T \in L(\mathcal{H}): E(T(x+\delta x))=x$ for every $x \in \mathcal{T}\}$. Then $T \in \mathcal{U}$ is called an approximation process over $\mathcal{T}$ if $E\|T \delta x\|^{2} \leq E\|U \delta x\|^{2}$ for every $U \in \mathcal{U}$.

Since $E(T(x+\delta x))=\int_{\Omega}(T x+T \delta x(\omega, \cdot)) d P(\omega)=T x$, because $E(T \delta x)$ $=0$ (see $[17$, Lemma 3]), every element of $\mathcal{U}$ satisfies $T x=x$ for every $x \in \mathcal{T}$, or, what is the same, $\mathcal{T} \subseteq N(I-T)$. The quantity $E\left(\|T \delta x\|^{2}\right)$ is related to the variance of $\delta x$. In fact, if $A \in L(\mathcal{H})^{+}$is the variance operator of $\delta x$, then $E\|T \delta x\|^{2}=\operatorname{Tr}\left(T A T^{*}\right.$ ) (see [17, Lemma 3]). The following theorem, due to Sard, gives a characterization of approximation processes.

Theorem ([17, Theorem 1]). Let $A \in L(\mathcal{H})^{+}$be the variance operator of a stochastic process $\delta x$ and $\mathcal{S}$ be a closed subspace of $\mathcal{H}$. Then $T \in L(\mathcal{H})$ is an approximation process over $\mathcal{S}^{\perp}$ with variance $A$ if and only if $R\left(A T^{*}\right) \subseteq$ $\mathcal{S}^{\perp} \subseteq N(I-T)$. Moreover, if $N(A) \cap \mathcal{S}=\{0\}$, then there exists a unique approximation process $T$.

Based on this theorem we state the following definition of a generalized approximation process, in which we do not constrain $A \in L(\mathcal{H})^{+}$to be a trace class operator.

Definition 3.2. Given $A \in L(\mathcal{H})^{+}$and $\mathcal{S}$ a closed subspace of $\mathcal{H}$. Then $T \in L(\mathcal{H})$ is an $A$-approximation process over $\mathcal{S}^{\perp}$ if $R\left(A T^{*}\right) \subseteq \mathcal{S}^{\perp} \subseteq$ $N(I-T)$.

Sard proved [17, Theorem 2] that a necessary and sufficient condition for the existence of an $A$-approximation process over $\mathcal{S}^{\perp}$ is that there exists 
a companion of $A$ relative to $\mathcal{S}^{\perp}$, or equivalently, by Proposition 2.5 , that the pair $(A, \mathcal{S})$ is compatible. This suggests a relationship between the set of $A$-selfadjoint projections with range $\mathcal{S}$ and the $A$-approximation processes over $\mathcal{S}$.

For $A \in L(\mathcal{H})^{+}$and $\mathcal{S}^{\perp}$ a closed subspace, let $\mathcal{P}_{*}\left(A, \mathcal{S}^{\perp}\right)=\{Q \in \mathcal{Q}$ : $\left.R(Q)=\mathcal{S}^{\perp}, A Q^{*}=Q A\right\}$.

REMARK 3.3. Observe that $\mathcal{P}_{*}\left(A, \mathcal{S}^{\perp}\right)$ is an affine manifold, possibly void: in fact, $Q \in \mathcal{P}_{*}\left(A, \mathcal{S}^{\perp}\right)$ if and only if $I-Q^{*} \in \mathcal{P}(A, \mathcal{S})$. Then by $(2.3)$, $\mathcal{P}_{*}\left(A, \mathcal{S}^{\perp}\right)=\left(I-P_{A, \mathcal{S}}^{*}\right)+L\left(N(A) \cap \mathcal{S}, \mathcal{S}^{\perp}\right)$. This manifold is contained in the set of $A$-approximation processes on $\mathcal{S}^{\perp}$, as shown by the following theorem.

Theorem 3.4. Let $A \in L(\mathcal{H})^{+}$and $\mathcal{S}$ be a closed subspace of $\mathcal{H}$. Let $\mathcal{A}$ be the set of $A$-approximation processes over $\mathcal{S}^{\perp}$. Then $\mathcal{A}$ is not empty if and only if the pair $(A, \mathcal{S})$ is compatible. In this case,

$$
\mathcal{A}=\mathcal{P}_{*}\left(A, \mathcal{S}^{\perp}\right)+L(\mathcal{N}, \mathcal{S}) .
$$

Proof. Suppose that $T \in L(\mathcal{H})$ is an $A$-approximation process over $\mathcal{S}^{\perp}$; then $R\left(A T^{*}\right) \subseteq N(I-T)$ so that $A T^{*}=T A T^{*}=T A$. Therefore $T$ is $A$-selfadjoint. From $R\left(A T^{*}\right) \subseteq \mathcal{S}^{\perp}$, we conclude that $P_{\mathcal{S}} A T^{*}=0=T A P_{\mathcal{S}}$. From $\mathcal{S}^{\perp} \subseteq N(I-T)$, it follows that $T=P_{\mathcal{S}^{\perp}}+T P_{\mathcal{S}}$, and so $I-T^{*}=$ $P_{\mathcal{S}}-P_{\mathcal{S}} T^{*}=P_{\mathcal{S}}-P_{\mathcal{S}} T^{*} P_{\mathcal{S}}-P_{\mathcal{S}} T^{*} P_{\mathcal{S}^{\perp}}$. Let $Q=P_{\mathcal{S}}-P_{\mathcal{S}} T^{*} P_{\mathcal{S}^{\perp}}$; it is easy to check that $Q$ is a projection with range $\mathcal{S}$. Also, from $A T^{*}=A P_{\mathcal{S}^{\perp}}+A P_{\mathcal{S}} T^{*}$, it follows that $A T^{*} P_{\mathcal{S}}=T A P_{\mathcal{S}}=0=A P_{\mathcal{S}} T^{*} P_{\mathcal{S}}$. Finally, observing that $A\left(I-T^{*}\right)=(I-T) A$, and that $I-T^{*}=Q-P_{\mathcal{S}} T^{*} P_{\mathcal{S}}$, we infer that $Q \in \mathcal{P}(A, \mathcal{S})$. Therefore $I-Q+W=T^{*}$ with $W=P_{\mathcal{S}} T^{*} P_{\mathcal{S}} \in L(\mathcal{S}, \mathcal{N})$ and $Q \in \mathcal{P}(A, \mathcal{S})$.

Conversely, if $T^{*}=I-Q+W$ with $Q \in \mathcal{P}(A, \mathcal{S})$ and $W \in L(\mathcal{S}, \mathcal{N})$, then it is easy to see that $T^{*}$ is $A$-selfadjoint. Moreover, $R\left(A T^{*}\right)=R(A(I-Q))=$ $R\left(\left(I-Q^{*}\right) A\right) \subseteq R\left(I-Q^{*}\right)=\mathcal{S}^{\perp}$ and $(I-T) P_{\mathcal{S}^{\perp}}=\left(Q^{*}-W^{*}\right) P_{\mathcal{S}^{\perp}}=0$, so that $\mathcal{S}^{\perp} \subseteq N(I-T)$.

An alternative characterization of $\mathcal{A}$ is given by

$$
\mathcal{A}=\left\{T \in L(\mathcal{H}): T=I-P_{A, \mathcal{S}}^{*}+W \text { for some } W \in L(\mathcal{N}, \mathcal{H})\right\} .
$$

This follows from the theorem above and (2.3).

4. Weighted least squares processes. Let $A \in L(\mathcal{H})^{+}, \mathcal{S}$ be a closed subspace of $\mathcal{H}$ and $y \in \mathcal{H}$. Any $u \in \mathcal{S}$ such that

$$
\|y-u\|_{A}=\min _{x \in \mathcal{S}}\|y-x\|_{A}
$$

is called a weighted least squares approximation of $y$ in $\mathcal{S}$ (with weight $A$ ) (hereafter, $A$-WLSA). 
In [17], the problem of finding an operator $T \in L(\mathcal{H})$ which assigns to each $y \in \mathcal{H}$ an $A$-WLSA is studied. Such an operator is called a weighted least squares process with weight $A$ ( $A$-WLSP).

Definition 4.1. Let $A \in L(\mathcal{H})^{+}$and $\mathcal{S}$ be a closed subspace of $\mathcal{H}$. Then $T \in L(\mathcal{H}, \mathcal{S})$ is an $A$-weighted least squares process ( $A$-WLSP) on $\mathcal{S}$ if for every $y \in \mathcal{H},\|y-T y\|_{A} \leq\|y-s\|_{A}$ for every $s \in \mathcal{S}$.

If the weight $A$ is proper on $\mathcal{S}$, Sard proved that there exists a unique $A$-WLSP and it is an $A$-selfadjoint projection. Later, the same problem, with different motivations than those of Sard, was studied in [7] and [10] under the compatibility hypothesis; it was shown that the compatibility of the pair $(A, \mathcal{S})$ is not only sufficient but also necessary for the existence of an $A$-WLSP. We summarize some of these results, more precisely [7, Proposition 4.4] and [10, Theorem 3.2], in the following statement. Notice, however, that we use the notation of the present paper, which is essentially that of Sard.

Theorem 4.2. Let $A \in L(\mathcal{H})^{+}$and $\mathcal{S}$ be a closed subspace of $\mathcal{H}$. The following conditions hold:

(1) There exists an $A$-WLSA on $\mathcal{S}$, for every $y \in \mathcal{H}$, if and only if the pair $(A, \mathcal{S})$ is compatible. In that case, it is unique if and only if $\mathcal{N}=\{0\}$.

(2) $u \in \mathcal{S}$ is an $A-W L S A$ of $y$ if and only if $y-u \in A(\mathcal{S})^{\perp}$.

(3) Every $Q \in \mathcal{P}(A, \mathcal{S})$ is an $A$-WLSP on $\mathcal{S}$.

Corollary 4.3. There exists an $A-W L S A$ on $\mathcal{S}$, for every $y \in \mathcal{S}^{\perp}$, if and only if the pair $(A, \mathcal{S})$ is compatible.

Proof. Suppose that, for every $\tilde{y} \in \mathcal{S}^{\perp}$, there exists an $A$-WLSA on $\mathcal{S}$. Given $y \in \mathcal{H} \backslash \mathcal{S}$, let $z_{0}$ be the $A$-WLSA of $P_{\mathcal{S}^{\perp}} y$. Then

$$
\left\|z_{0}-P_{\mathcal{S}^{\perp}} y\right\|_{A} \leq\left\|z-P_{\mathcal{S}^{\perp}} y\right\|_{A} \quad \text { for every } z \in \mathcal{S} .
$$

Let $x_{0}=z_{0}+P_{\mathcal{S}} y$ and $x=z+P_{\mathcal{S}} y$. Then

$$
\left\|y-x_{0}\right\|_{A} \leq\|y-x\|_{A} .
$$

Since (4.2) holds for any $z \in \mathcal{S}$, we see that $x$ is an arbitrary vector in $\mathcal{S}$, thus $x_{0}$ is an $A$-WLSP for $y \in \mathcal{H} \backslash \mathcal{S}$. Furthermore, if $y \in \mathcal{S}$, then $x_{0}=y$ is an $A$-WLSA on $\mathcal{S}$, because $A \in L(\mathcal{H})^{+}$. Hence, there exists an $A$-WLSA for every $y \in \mathcal{H}$, thus, by Theorem $4.2,(A, \mathcal{S})$ is compatible.

The converse follows directly from Theorem 4.2.

By Proposition 2.7, it is clear that the condition of $A$ being proper on $\mathcal{S}$ is sufficient but not necessary for the existence and uniqueness of $A$-WSLP. Furthermore, $(3)$ shows that every element in $\mathcal{P}(A, \mathcal{S})$ is an $A$-WLSP. Notice, however, that an $A$-WLSP may not be a projection, as shown by the next proposition. 
Proposition 4.4. Let $A \in L(\mathcal{H})^{+}$and $\mathcal{S}$ be a closed subspace of $\mathcal{H}$ such that the pair $(A, \mathcal{S})$ is compatible. The operator $W \in L(\mathcal{H})$ is an $A$-WLSP if and only if $W \in P_{A, \mathcal{S}}+L(\mathcal{H}, \mathcal{N})$.

Proof. Suppose that $W \in L(\mathcal{H})$ is an $A$-WLSP. Then, by Theorem $4.2(2)$, for every $y \in \mathcal{H}$ we have $y-W y \in A(\mathcal{S})^{\perp}$, i.e.

$$
\langle W y-y, z\rangle_{A}=0 \quad \text { for every } z \in \mathcal{S} \text {. }
$$

Hence,

$$
0=\left\langle W y-y, P_{A, \mathcal{S}} x\right\rangle_{A}=\left\langle W y-P_{A, \mathcal{S}} y, x\right\rangle_{A} \quad \text { for every } x \in \mathcal{H},
$$

because $R(W) \subseteq \mathcal{S}$, and $L=W-P_{A, \mathcal{S}} \in L(\mathcal{H}, \mathcal{N})$.

Conversely, suppose that $W=P_{A, \mathcal{S}}+L$, where $L \in L(\mathcal{H}, \mathcal{N})$; then, by Proposition 4.2 and the comments above, it is easy to see that, given $y \in \mathcal{H}$, $\langle W y-y, z\rangle_{A}=\left\langle P_{A, \mathcal{S}} y-y, z\right\rangle_{A}=0$ for every $z \in \mathcal{S}$.

An interesting problem, which naturally occurs in some signal processing applications, is the following: given a closed subspace $\mathcal{S}$ and $A, B \in L(\mathcal{H})^{+}$, find a $B$-approximation process over $\mathcal{S}$ which is also an $A$-WLSP on $\mathcal{S}$. In [17], this problem has been studied under the assumption that the weight $A$ is proper on $\mathcal{S}$. In this section we study this problem under the assumption that the pair $(A, \mathcal{S})$ is compatible.

The next result shows that the set of $B$-approximation processes over $\mathcal{S}$ which are also $A$-WLSP on $\mathcal{S}$ is the intersection of the affine manifolds $\mathcal{P}_{*}(B, \mathcal{S})$ and $\mathcal{P}(A, \mathcal{S})$. Notice that if $T$ is a $B$-approximation process and also an $A$-WLSP on $\mathcal{S}$, then $T$ is a projection. If $\mathcal{N}=\{0\}$, in particular if $A$ is proper on $\mathcal{S}$, the problem of finding $B$-approximation processes which are also $A$-WLSP on $\mathcal{S}$ reduces to checking if $P_{A, \mathcal{S}} \in \mathcal{P}_{*}(B, \mathcal{S})$.

Observe that, by Theorems 3.4 and 4.2 , the compatibility of the pairs $(A, \mathcal{S})$ and $\left(B, \mathcal{S}^{\perp}\right)$ is necessary for the existence of $B$-approximation processes which are also $A$-WLSP over $\mathcal{S}$.

Lemma 4.5. Let $A, B \in L(\mathcal{H})^{+}$and $\mathcal{S}$ be a closed subspace such that the pairs $(A, \mathcal{S})$ and $\left(B, \mathcal{S}^{\perp}\right)$ are compatible. Then $T \in L(\mathcal{H})$ is a $B$-approximation process over $\mathcal{S}$ and also an $A-W L S P$ on $\mathcal{S}$ if and only if $T \in \mathcal{P}_{*}(B, \mathcal{S}) \cap$ $\mathcal{P}(A, \mathcal{S})$.

Proof. If $T \in \mathcal{P}(A, \mathcal{S}) \cap \mathcal{P}_{*}(B, \mathcal{S})$, then by Proposition 4.4 and Theorem 3.4, it follows that $T$ is an $A$-WLSP and also an approximation process.

Conversely, suppose that $T$ is a $B$-approximation process over $\mathcal{S}$. By Theorem 3.4, $T=(I-Q)^{*}+Z$, where $Q \in \mathcal{P}\left(B, \mathcal{S}^{\perp}\right)$ and $Z \in L(N(B) \cap$ $\left.\mathcal{S}^{\perp}, \mathcal{S}^{\perp}\right)$. If $T$ is an $A$-WLSP in $\mathcal{S}$ then $R(T) \subseteq \mathcal{S}$, which implies that $\mathcal{S}^{\perp} \subseteq R(T)^{\perp}=N\left(T^{*}\right)$. Since $T^{*}=(I-Q)+Z$ we have $Z x=0$ for every $x \in \mathcal{S}^{\perp}$, i.e., $T=(I-Q)^{*} \in \mathcal{P}_{*}(B, \mathcal{S})$. Also, by Proposition $4.4, T=P+R$ 
with $P \in \mathcal{P}(A, \mathcal{S})$ and $R \in L(\mathcal{S}, N(A) \cap \mathcal{S})$. Since $T$ is a projection with range $\mathcal{S}$, it is easy to see that $R=0$, and so $T \in \mathcal{P}(A, \mathcal{S}) \cap \mathcal{P}_{*}(B, \mathcal{S})$.

The next theorem states necessary and sufficient conditions for the nonemptiness of $\mathcal{P}(A, \mathcal{S}) \cap \mathcal{P}_{*}(B, \mathcal{S})$, and hence for the existence of approximation processes which are also $A$-WLSP on $\mathcal{S}$.

Theorem 4.6. Let $A, B \in L(\mathcal{H})^{+}$and $\mathcal{S}$ be a closed subspace of $\mathcal{H}$. Let $\mathcal{N}=N(A) \cap \mathcal{S}$ and $\mathcal{M}=N(B) \cap \mathcal{S}^{\perp}$. The following conditions are equivalent:

(1) $\mathcal{P}(A, \mathcal{S}) \cap \mathcal{P}_{*}(B, \mathcal{S}) \neq \emptyset$.

(2) $(A, \mathcal{S})$ is compatible, $A B\left(\mathcal{S}^{\perp}\right) \subseteq \mathcal{S}^{\perp}$ and $c_{0}\left(\mathcal{N}, B\left(\mathcal{S}^{\perp}\right)\right)<1$.

(3) $\left(B, \mathcal{S}^{\perp}\right)$ is compatible, $B A(\mathcal{S}) \subseteq \mathcal{S}$ and $c_{0}(\mathcal{M}, A(\mathcal{S}))<1$.

Proof. $(1) \Rightarrow(2)$ : Suppose that $Q=P_{\mathcal{S} / / \mathcal{T}} \in \mathcal{P}(A, \mathcal{S}) \cap \mathcal{P}_{*}(B, \mathcal{S})$. Since $Q \in \mathcal{P}(A, \mathcal{S})$, we have $\mathcal{T} \subseteq A^{-1}\left(\mathcal{S}^{\perp}\right)$. Analogously, $Q^{*}=P_{\mathcal{T}^{\perp} / / \mathcal{S}^{\perp}} \in$ $P\left(B, \mathcal{T}^{\perp}\right)$, because $Q \in \mathcal{P}_{*}(B, \mathcal{S})$; therefore $\mathcal{S}^{\perp} \subseteq B^{-1}(\mathcal{T})$. Then $B\left(\mathcal{S}^{\perp}\right) \subseteq$ $\mathcal{T} \subseteq A^{-1}\left(\mathcal{S}^{\perp}\right)$, i.e. $A B\left(\mathcal{S}^{\perp}\right) \subseteq \mathcal{S}^{\perp}$. Furthermore, $B\left(\mathcal{S}^{\perp}\right) \cap \mathcal{N} \subseteq \mathcal{T} \cap \mathcal{N}=\{0\}$, so $c\left(B\left(\mathcal{N}, \mathcal{S}^{\perp}\right)\right)=c_{0}\left(\mathcal{N}, B\left(\mathcal{S}^{\perp}\right)\right)$ and $c\left(\mathcal{N}, B\left(\mathcal{S}^{\perp}\right)\right)<c(\mathcal{N}, \mathcal{T})<c(\mathcal{S}, \mathcal{T})<1$.

$(2) \Rightarrow(1)$ : Suppose $A B\left(\mathcal{S}^{\perp}\right) \subseteq \mathcal{S}^{\perp}, \mathcal{N} \cap B\left(\mathcal{S}^{\perp}\right)=\{0\}$ and $c_{0}\left(\mathcal{N}, B\left(\mathcal{S}^{\perp}\right)\right)$ $<1$. Then $B\left(\mathcal{S}^{\perp}\right) \dot{+} \mathcal{N}$ is a closed subspace of $A^{-1}\left(\mathcal{S}^{\perp}\right)$. Let $\mathcal{W}$ be a closed subspace with $B\left(\mathcal{S}^{\perp}\right) \dot{+} \mathcal{N} \dot{+} \mathcal{W}=A^{-1}\left(\mathcal{S}^{\perp}\right)$. Let $\mathcal{T}=B\left(\mathcal{S}^{\perp}\right) \dot{+} \mathcal{W} \subseteq A^{-1}\left(\mathcal{S}^{\perp}\right)$; observe that $\mathcal{H}=\mathcal{S} \dot{+} \mathcal{T}$, and define $Q=P_{\mathcal{S} / / \mathcal{T}}$. Since $N(Q) \subseteq A^{-1}\left(\mathcal{S}^{\perp}\right)$, we have $Q \in \mathcal{P}(A, \mathcal{S})$ (see Preliminaries). Furthermore, since $B\left(\mathcal{S}^{\perp}\right) \subseteq \mathcal{T}$, it follows that $N\left(Q^{*}\right)=\mathcal{S}^{\perp} \subseteq B^{-1}(\mathcal{T})$, i.e., $Q^{*}$ is a $B$-selfadjoint projection. Thus $Q \in \mathcal{P}(A, \mathcal{S}) \cap \mathcal{P}_{*}(B, \mathcal{S})$.

$(1) \Leftrightarrow(3)$ follows analogously.

Notice that the compatibility of the pair $(A, \mathcal{S})$ and the conditions $A B\left(\mathcal{S}^{\perp}\right) \subseteq \mathcal{S}^{\perp}$ and $c_{0}\left(\mathcal{N}, B\left(\mathcal{S}^{\perp}\right)\right)<1$ imply the compatibility of the pair $\left(B, \mathcal{S}^{\perp}\right)$.

If $\mathcal{N}=\{0\}$, the hypotheses which guarantee the existence of $B$-approximation processes over $\mathcal{S}$ which are also $A$-WLSP on $\mathcal{S}$ reduce to $A B\left(\mathcal{S}^{\perp}\right) \subseteq$ $\mathcal{S}^{\perp}$. Then we recover, under the compatibility hypothesis, the result proved in [17, Theorem 4] for an $A \in L(\mathcal{H})^{+}$which is proper on $\mathcal{S}$.

The next result gives alternative conditions for the existence of $B$-approximation processes which are also $A$-WLSP on $\mathcal{S}$. This will be useful to construct such operators. Recall that $P_{\mathcal{T}}$ is the orthogonal projection onto $\mathcal{T}$.

Lemma 4.7. Suppose that $A, B \in L(\mathcal{H})^{+}$and $\mathcal{S}$ is a closed subspace such that the pairs $(A, \mathcal{S})$ and $\left(B, \mathcal{S}^{\perp}\right)$ are compatible. Let $P \in \mathcal{P}(A, \mathcal{S})$, $Q \in \mathcal{P}\left(B, \mathcal{S}^{\perp}\right), \mathcal{N}=N(A) \cap \mathcal{S}$ and $\mathcal{M}=N(B) \cap \mathcal{S}^{\perp}$. Then the following conditions are equivalent: 
(1) $\mathcal{P}(A, \mathcal{S}) \cap \mathcal{P}_{*}(B, \mathcal{S}) \neq \emptyset$,

(2) $A\left(I-P-Q^{*}\right) P_{\mathcal{M}^{\perp}}=0$,

(3) $B\left(I-P^{*}-Q\right) P_{\mathcal{N} \perp}=0$.

Proof. (1) $\Rightarrow(2)$ : Let $T \in \mathcal{P}(A, \mathcal{S}) \cap \mathcal{P}_{*}(B, \mathcal{S})$. Then, by Lemma $4.5, T$ is an $A$-WLSP over $\mathcal{S}$ and a $B$-approximation process over $\mathcal{S}$. By $(2.3)$ and Remark 3.3,

$$
T=P+L_{1}=\left(I-Q^{*}\right)+L_{2},
$$

where $L_{1} \in L\left(\mathcal{S}^{\perp}, \mathcal{N}\right)$ and $L_{2} \in L(\mathcal{M}, \mathcal{S})$.

Thus $I-Q^{*}-P=L_{1}-L_{2}$. Let $Z=I-Q^{*}-P$; it is easy to see that $R(Z) \subseteq \mathcal{S}$ and $Z\left(\mathcal{M}^{\perp}\right) \subseteq \mathcal{N}$ (because $\mathcal{M}^{\perp} \subseteq N\left(L_{2}\right)$ ). Consequently, $A\left(I-Q^{*}-P\right) P_{\mathcal{M}^{\perp}}=0$.

$(2) \Rightarrow(1)$ : Suppose that $A\left(I-Q^{*}-P\right) P_{\mathcal{M}^{\perp}}=0$. Let $Z=I-Q^{*}-P$. Observe that $Z(\mathcal{S})=0$ and $R(Z) \subseteq \mathcal{S}$. Furthermore, $Z\left(\mathcal{M}^{\perp}\right) \subseteq \mathcal{N}$. Let $L_{1}=Z P_{\mathcal{M}^{\perp}}$ and $L_{2}=Z P_{\mathcal{M}}$.

Then $Z=L_{1}+L_{2}=I-Q^{*}-P$, or equivalently, $1-Q^{*}-L_{2}=P+L_{1}$. If $T=1-Q^{*}-L_{2}=P+L_{1}$, then $T \in \mathcal{P}(A, \mathcal{S}) \cap \mathcal{P}_{*}(B, \mathcal{S})$; in fact, $R\left(L_{1}\right) \subseteq \mathcal{N}$ and $L_{1}(\mathcal{S})=0$ (because $\mathcal{S} \subseteq \mathcal{M}^{\perp}$ and $Z(\mathcal{S})=0$ ) so that $L_{1} \in L\left(\mathcal{S}^{\perp}, \mathcal{N}\right)$. Also, $\mathcal{M}^{\perp} \subseteq N\left(L_{2}\right)$ and $R\left(L_{2}\right) \subseteq \mathcal{S}$, so $L_{2} \in L(\mathcal{M}, \mathcal{S})$.

Thus $\left(I-Q^{*}\right)-L_{2}=P+L_{1}$. Set $T=P+L_{1}=\left(I-Q^{*}\right)-L_{2}$. It follows that $T \in\left(P+L\left(\mathcal{S}^{\perp}, \mathcal{N}\right)\right) \cap\left(\left(I-Q^{*}\right)+L(\mathcal{S}, \mathcal{M})^{*}\right)$.

$(1) \Leftrightarrow(3)$ is analogous to $(1) \Leftrightarrow(2)$.

The following theorem gives a parametrization of the set of $B$-approximation processes which are also $A$-WLSP on $\mathcal{S}$, i.e., the set $\mathcal{P}(A, \mathcal{S}) \cap \mathcal{P}_{*}(B, \mathcal{S})$.

TheOREM 4.8. Let $A, B \in L(\mathcal{H})^{+}$and let $\mathcal{S}$ be a closed subspace such that $\mathcal{P}(A, \mathcal{S}) \cap \mathcal{P}_{*}(B, \mathcal{S}) \neq \emptyset$. Let $\mathcal{N}=N(A) \cap \mathcal{S}$ and $\mathcal{M}=N(B) \cap \mathcal{S}^{\perp}$. Then

$$
\mathcal{P}(A, \mathcal{S}) \cap \mathcal{P}_{*}(B, \mathcal{S})=\left(I-P_{B, \mathcal{S}^{\perp}}^{*}\right) P_{\mathcal{M}^{\perp}}+P_{A, \mathcal{S}} P_{\mathcal{M}}+L(\mathcal{M}, \mathcal{N}) .
$$

Proof. If $E=\left(I-P_{B, \mathcal{S}^{\perp}}^{*}\right) P_{\mathcal{M}^{\perp}}+P_{A, \mathcal{S}} P_{\mathcal{M}}+C$ for some $C \in L(\mathcal{M}, \mathcal{N})$, then

$$
\begin{aligned}
A E & =A\left(\left(I-P_{B, \mathcal{S}^{\perp}}^{*}\right) P_{\mathcal{M}^{\perp}}+P_{A, \mathcal{S}} P_{\mathcal{M}}\right) \\
& =A P_{A, \mathcal{S}}-A P_{A, \mathcal{S}} P_{\mathcal{M}^{\perp}}+A P_{\mathcal{M}^{\perp}}-A P_{B, \mathcal{S}^{\perp}}^{*} P_{\mathcal{M}^{\perp}}=A P_{A, \mathcal{S}},
\end{aligned}
$$

since, by Lemma 4.7, $A\left(I-P_{A, \mathcal{S}}-P_{B, \mathcal{S}^{\perp}}^{*}\right) P_{\mathcal{M}^{\perp}}=0$. Thus $E$ is $A$-selfadjoint. Furthermore, since $\mathcal{S} \subseteq \mathcal{M}^{\perp}$ and $C=C P_{\mathcal{M}}$, it follows that $E^{2}=E$. Moreover, it is easy to see that $E P_{\mathcal{S}}=P_{\mathcal{S}}$, so $E \in \mathcal{P}(A, \mathcal{S})$. Analogously, $E \in \mathcal{P}_{*}\left(B, \mathcal{S}^{\perp}\right)$. Therefore $E$ is a $B$-approximation process and also an $A$-WLSP on $\mathcal{S}$.

Conversely, let $Q \in \mathcal{P}(A, \mathcal{S}) \cap \mathcal{P}_{*}(B, \mathcal{S})$ and $C=Q-\left(I-P_{B, \mathcal{S}^{\perp}}^{*}\right) P_{\mathcal{M}^{\perp}-}$ $P_{A, \mathcal{S}} P_{\mathcal{M}}$. Then $C P_{\mathcal{M}^{\perp}}=Q P_{\mathcal{M}^{\perp}}-\left(I-P_{B, \mathcal{S}^{\perp}}^{*}\right) P_{\mathcal{M}^{\perp}}$. Since $Q \in \mathcal{P}_{*}\left(B, \mathcal{S}^{\perp}\right)$, it follows that $Q=\left(I-P_{B, \mathcal{S}^{\perp}}^{*}\right)+W$ with $W \in L(\mathcal{M}, \mathcal{S})$. Hence $Q P_{\mathcal{M}^{\perp}}=$ 
$\left(I-P_{B, \mathcal{S}^{\perp}}^{*}\right) P_{\mathcal{M}^{\perp}}$, so that $C P_{\mathcal{M}^{\perp}}=0$. Furthermore, since $Q \in \mathcal{P}(A, \mathcal{S})$, we have $Q=P_{A, \mathcal{S}}+\tilde{W}$ with $\tilde{W} \in L\left(\mathcal{S}^{\perp}, \mathcal{N}\right)$. Therefore, $C=C P_{\mathcal{M}}=$ $P_{A, \mathcal{S}} P_{\mathcal{M}}-\tilde{W} P_{\mathcal{M}}-P_{A, \mathcal{S}} P_{\mathcal{M}}$, so that $R(C) \subseteq \mathcal{N}$. Thus, $Q \in\left(I-P_{B, \mathcal{S}^{\perp}}^{*}\right) P_{\mathcal{M}^{\perp}}+$ $P_{A, \mathcal{S}} P_{\mathcal{M}}+L(\mathcal{M}, \mathcal{N})$

5. Curve fitting processes. Suppose that $\mathcal{H}=L^{2}(\mu)$ and $\delta x=$ $\delta x(t, \omega)$ is a stochastic process, as defined in Section 3. Given a linearly independent set $\left\{v_{n}\right\}_{n \in \mathcal{I}=\{1, \ldots, N\}}$ that spans a (finite-dimensional) subspace $\mathcal{S}$ of $\mathcal{H}$ and the variance operator of $\delta x, A \in L(\mathcal{H})^{+}$, Sard [17] studied the problem of finding $\left\{w_{n}\right\}_{n \in \mathcal{I}} \subseteq \mathcal{H}$ such that

$$
E\left(\sum_{n \in \mathcal{I}}\left\langle w_{n}, x+\delta x\right\rangle v_{n}\right)=x \quad \text { for every } x \in \mathcal{S},
$$

and minimize

$$
E\left(\sum_{n \in \mathcal{I}}\left|\left\langle w_{n}, \delta x\right\rangle\right|^{2}\right)
$$

In $[17$, Lemmas 15 and 16$]$, it was proved that

$$
E\left(\sum_{n \in \mathcal{I}}\left|\left\langle w_{n}, \delta x\right\rangle\right|^{2}\right)=\sum_{n \in \mathcal{I}}\left\langle w_{n}, w_{n}\right\rangle_{A}
$$

In this section we generalize this problem and study the existence of solutions under the assumption that $\left\{v_{n}\right\}_{n \in \mathcal{I} \subseteq \mathbb{Z}}$ is a frame for a (possibly infinite-dimensional) closed subspace $\mathcal{S}$ of $\mathcal{H}$. First, we introduce some definitions and results.

Definition 5.1. Let $\mathcal{S}$ be a closed subspace of $\mathcal{H}$. The set $\mathcal{V}=\left\{v_{n}\right\}_{n \in \mathcal{I}}$ $\subseteq \mathcal{S}$ is a frame for $\mathcal{S}$ if there exist constants $\gamma_{1}, \gamma_{2}>0$ such that

$$
\gamma_{1}\|x\|^{2} \leq \sum_{n \in \mathcal{I}}\left|\left\langle x, v_{n}\right\rangle\right|^{2} \leq \gamma_{2}\|x\|^{2} \quad \text { for every } x \in \mathcal{S} .
$$

If the set $\mathcal{V}=\left\{v_{n}\right\}_{n \in \mathcal{I}}$ is also linearly independent then it is called a Riesz basis of $\mathcal{S}$.

Let $\mathcal{S}$ be a closed subspace of $\mathcal{H}$ and let $\mathcal{V}=\left\{v_{n}\right\}_{n \in \mathcal{I}}$ be a frame for $\mathcal{S}$. Let $\mathcal{K}$ be a (separable) Hilbert space and $\mathcal{B}=\left\{e_{n}\right\}_{n \in \mathcal{I}}$ an orthonormal basis of $\mathcal{K}$. By (5.3), there exists a unique $F \in L(\mathcal{K}, \mathcal{H})$ such that $F e_{n}=v_{n}$ for every $n \in \mathcal{I}$. The triplet $(F, \mathcal{B}, \mathcal{K})$ is called the synthesis operator of $\mathcal{V}$, and the analysis operator of $\mathcal{V}$ is $F^{*} \in L(\mathcal{H}, \mathcal{K})$ given by $F^{*} x=\sum_{n \in \mathcal{I}}\left\langle x, v_{n}\right\rangle e_{n}$. The operator $T=F F^{*} \in L(\mathcal{H})$ does not depend on the synthesis operator $F$ but only on the frame $\mathcal{V}=\left\{v_{n}\right\}_{n \in \mathcal{I}}$ (see [5]), i.e., if $\left(F_{1}, \mathcal{K}_{1}, \mathcal{B}_{1}\right)$ is another synthesis operator for $\mathcal{V}$ then $T=F_{1} F_{1}^{*}=F F^{*} ; T$ is called the frame operator of $\mathcal{V}$. 
The restriction of $T$ to the subspace $\mathcal{S}$ is invertible; moreover, from (5.3), $\gamma_{1} P_{\mathcal{S}} \leq P_{\mathcal{S}} T P_{\mathcal{S}} \leq \gamma_{2} P_{\mathcal{S}}$ and so $\left(1 / \gamma_{1}\right) P_{\mathcal{S}} \leq P_{\mathcal{S}} T^{\dagger} P_{\mathcal{S}} \leq\left(1 / \gamma_{2}\right) P_{\mathcal{S}}$, where $T^{\dagger}$ denotes the Moore-Penrose inverse of $T$.

Based on the above definitions, the problem stated in (5.1) and (5.2) and solved by Sard can be rewritten as follows. Let $\mathcal{V}=\left\{v_{n}\right\}_{n \in \mathcal{I}=\{1, \ldots, N\}}$ be a basis of a closed subspace $\mathcal{S}$ and $\left(F,\left\{e_{n}\right\}_{n \in \mathcal{I}}, \mathbb{C}^{N}\right)$ its synthesis operator (where $\left\{e_{n}\right\}_{n \in \mathcal{I}}$ is the canonical basis in $\mathbb{C}^{N}$ ). Find $G_{0} \in L\left(\mathbb{C}^{N}, \mathcal{H}\right)$ such that

(1) $G_{0}$ satisfies $F G_{0}^{*} P_{\mathcal{S}}=P_{\mathcal{S}}$,

(2) $\sum_{n}\left\langle G_{0} e_{k}, G_{0} e_{k}\right\rangle_{A} \leq \sum_{n}\left\langle G e_{k}, G e_{k}\right\rangle_{A}$ for every $G \in L\left(\mathbb{C}^{N}, \mathcal{H}\right)$ such that $F G^{*} P_{\mathcal{S}}=P_{\mathcal{S}}$.

Notice that $\sum_{n}\left\langle G e_{k}, G e_{k}\right\rangle_{A}=\operatorname{Tr}\left(G^{*} A G\right)$. Based on this reformulation of the original problem, we give the following definition.

Definition 5.2. Let $\mathcal{H}$ and $\mathcal{K}$ be two (separable) Hilbert spaces, $A \in$ $L(\mathcal{H})^{+}$a trace class operator and $\mathcal{S}$ a closed subspace of $\mathcal{H}$. Let $\mathcal{V}=\left\{v_{n}\right\}_{n \in \mathcal{I}}$ be a frame for $\mathcal{S}$ and $(F, \mathcal{B}, \mathcal{K})$ a synthesis operator of $\mathcal{V}$. Then $F G_{0}^{*}$ is an $A$-curve fitting process on $\mathcal{S}$ if

$$
\operatorname{Tr}\left(G_{0}^{*} A G_{0}\right)=\min \left\{\operatorname{Tr}\left(G^{*} A G\right): G \in L(\mathcal{K}, \mathcal{H}), F G^{*} P_{\mathcal{S}}=P_{\mathcal{S}}\right\} .
$$

As we will show later, the problem of finding such a $G_{0} \in L(\mathcal{K}, \mathcal{H})$ is related to an abstract spline problem. We first characterize the set of (bounded linear) operators satisfying condition (1).

Lemma 5.3. Let $\mathcal{V}=\left\{v_{n}\right\}_{n \in \mathcal{I}}$ be a frame for a closed subspace $\mathcal{S}$, let $(F, \mathcal{B}, \mathcal{K})$ be a synthesis operator for $\mathcal{V}$, and $T=F F^{*}$. Then $G \in L(\mathcal{K}, \mathcal{H})$ satisfies $F G^{*} P_{\mathcal{S}}=P_{\mathcal{S}}$ if and only if $G P_{N(F)^{\perp}}=T^{\dagger} F+L\left(N(F)^{\perp}, \mathcal{S}^{\perp}\right)$.

Proof. Since $R(T)=R(F)=\mathcal{S}$, it follows that $P_{\mathcal{S}}=T T^{\dagger}$. Suppose that $G \in L(\mathcal{K}, \mathcal{H})$ satisfies $F G^{*} P_{\mathcal{S}}=P_{\mathcal{S}}$. Then $F\left(F^{*} T^{\dagger}-G^{*}\right) F=0$. Since $R\left(F^{*}\right)$ is closed, it follows that $F^{*}\left(T^{\dagger} F-G\right) P_{N(F)^{\perp}}=0$, so $R\left(\left(T^{\dagger} F-G\right) P_{N(F)^{\perp}}\right) \subseteq$ $N\left(F^{*}\right)=\mathcal{S}^{\perp}$. Let $W=G P_{N(F)^{\perp}}-T^{\dagger} F P_{N(F)^{\perp}}=G P_{N(F)^{\perp}}-T^{\dagger} F$. Then $R(W) \subseteq \mathcal{S}^{\perp}$ and $N(F) \subseteq N(W)$, thus $G P_{N(F)^{\perp}} \in T^{\dagger} F+L\left(N(F)^{\perp}, \mathcal{S}^{\perp}\right)$.

Conversely, suppose that $G P_{N(F)^{\perp}}=T^{\dagger} F+W$ with $W \in L\left(N(F)^{\perp}, \mathcal{S}^{\perp}\right)$. Then $F^{*}\left(T^{\dagger} F-G\right) P_{N(F)^{\perp}}=0$, which implies that $P_{N(F)^{\perp}}\left(G^{*}-F^{*} T^{\dagger}\right) F=0$, so $R\left(\left(G^{*}-F^{*} T^{\dagger}\right) F\right) \subseteq N(F)$, i.e., $F\left(G^{*}-F^{*} T^{\dagger}\right) F=0$, so that $F G^{*} P_{\mathcal{S}}$ $=P_{\mathcal{S}}$.

If $\mathcal{V}=\left\{v_{n}\right\}_{n \in \mathcal{I}}$ is a Riesz basis of $\mathcal{S}$, then $N(F)=0$ and Lemma 5.3 asserts that $F G^{*} P_{\mathcal{S}}=P_{\mathcal{S}}$ if and only if $G=T^{\dagger} F+L\left(\mathcal{K}, \mathcal{S}^{\perp}\right)$. 
Given two Hilbert spaces $\mathcal{H}$ and $\mathcal{K}, C \in L(\mathcal{H}, \mathcal{K}), \mathcal{M}$ a closed subspace of $\mathcal{H}$ and $\xi \in \mathcal{H}$, an abstract spline interpolant to $\xi$ is any element in the set

$$
\operatorname{sp}(C, \mathcal{M}, \xi)=\left\{\eta \in \xi+\mathcal{M}:\|C \eta\|=\min _{\sigma \in \mathcal{M}}\|C(\xi+\sigma)\|\right\} .
$$

See $[6,11,15]$.

The following result [10, Theorem 3.2] establishes the relation between compatibility and the existence of abstract spline interpolants.

Theorem 5.4. Let $C \in L(\mathcal{H}, \mathcal{K})$ and $\mathcal{M}$ be a closed subspace of $\mathcal{H}$. The set $\operatorname{sp}(C, \mathcal{M}, \xi)$ is not empty, for every $\xi \in \mathcal{H}$, if and only if the pair $\left(C^{*} C, \mathcal{M}\right)$ is compatible. Moreover, in this case, $\operatorname{sp}(C, \mathcal{M}, \xi)=\{(I-Q) \xi$ : $\left.Q \in \mathcal{P}\left(C^{*} C, \mathcal{M}\right)\right\}$.

Based on this theorem, we can give conditions for the existence of $A$-curve fitting processes.

TheOREM 5.5. Let $A \in L(\mathcal{H})^{+}$be a trace class operator, $\mathcal{S}$ a closed subspace of $\mathcal{H}, \mathcal{V}=\left\{v_{n}\right\}_{n \in \mathcal{I}}$ a frame for $\mathcal{S},\left(F, \mathcal{B}=\left\{e_{n}\right\}_{n \in \mathcal{I}}, \mathcal{K}\right)$ the synthesis operator of $\mathcal{V}$, and $T=F F^{*}$. Then there exists an $A$-curve fitting process on $\mathcal{S}$ if and only if the pair $\left(A, \mathcal{S}^{\perp}\right)$ is compatible. Moreover, in this case, given $Q \in \mathcal{P}\left(A, \mathcal{S}^{\perp}\right), G_{0}=(I-Q) T^{\dagger} F$ is such that $F G_{0}$ is an $A$-curve fitting process on $\mathcal{S}$.

Proof. Let $\mathcal{I}_{1}, \mathcal{I}_{2} \subseteq \mathcal{I}$ be such that $\mathcal{I}_{1} \cup \mathcal{I}_{2}=\mathcal{I},\left\{\varepsilon_{n}\right\}_{n \in \mathcal{I}_{1}}$ is an orthonormal basis of $N(F)$ and $\left\{\varepsilon_{n}\right\}_{n \in \mathcal{I}_{2}}$ is an orthonormal basis of $N(F)^{\perp}$.

Let $Q \in \mathcal{P}\left(A, \mathcal{S}^{\perp}\right)$. Then applying Lemma 5.3 it is easy to see that $G_{0}=(I-Q) T^{\dagger} F \in L(\mathcal{K}, \mathcal{H})$ satisfies $F G_{0}^{*} P_{\mathcal{S}}=P_{\mathcal{S}}$.

Suppose that $G \in L(\mathcal{K}, \mathcal{H})$ satisfies $F G^{*} P_{\mathcal{S}}=P_{\mathcal{S}}$. Then, by Lemma 5.3,

$$
G P_{N(F)^{\perp} \varepsilon_{n}} \in T^{\dagger} F \varepsilon_{n}+\mathcal{S}^{\perp}
$$

and by Theorem 5.4, $\left\|A^{1 / 2}(I-Q) T^{\dagger} F \varepsilon_{n}\right\| \leq\left\|A^{1 / 2} h\right\|$ for every $h \in T^{\dagger} F \varepsilon_{n}$ $+\mathcal{S}^{\perp}$. Hence

$$
\begin{aligned}
\operatorname{Tr}\left(G_{0}^{*} A G_{0}\right) & =\sum_{n \in \mathcal{I}}\left\|G_{0} \varepsilon_{n}\right\|_{A}^{2}=\sum_{n \in \mathcal{I}}\left\|(I-Q) T^{\dagger} F \varepsilon_{n}\right\|_{A}^{2} \\
& =\sum_{n \in \mathcal{I}_{2}}\left\|(I-Q) T^{\dagger} F \varepsilon_{n}\right\|_{A}^{2} \leq \sum_{n \in \mathcal{I}_{2}}\left\|G P_{N(F)^{\perp}} \varepsilon_{n}\right\|_{A}^{2}=\sum_{n \in \mathcal{I}_{2}}\left\|G \varepsilon_{n}\right\|_{A}^{2} \\
& \leq \sum_{n \in \mathcal{I}_{2}}\left\|G \varepsilon_{n}\right\|_{A}^{2}+\sum_{n \in \mathcal{I}_{1}}\left\|G \varepsilon_{n}\right\|_{A}^{2}=\operatorname{Tr}\left(G^{*} A G\right),
\end{aligned}
$$

since $\sum_{n \in \mathcal{I}_{1}}\left\|G \varepsilon_{n}\right\|_{A} \geq 0$.

Conversely, if $F G^{*} P_{\mathcal{S}}=P_{\mathcal{S}}$ then, by Lemma 5.3, $G=T^{\dagger} F+W+R$ for some $W \in L\left(N(F)^{\perp}, \mathcal{S}^{\perp}\right)$ and $R \in L(N(F), \mathcal{H})$. Suppose that $G_{0}=T^{\dagger} F+$ $W_{0}+R_{0}$, with $W_{0} \in L\left(N(F)^{\perp}, \mathcal{S}^{\perp}\right)$ and $R_{0} \in L(N(F), \mathcal{H})$, is an $A$-curve 
fitting process. Then, for every $W \in L\left(N(F)^{\perp}, \mathcal{S}^{\perp}\right)$ and $R \in L(N(F), \mathcal{H})$,

$$
\sum_{n}\left\|G_{0} \varepsilon_{n}\right\|_{A}^{2} \leq \sum_{n}\left\|\left(T^{\dagger} F+W+R\right) \varepsilon_{n}\right\|_{A}^{2} .
$$

Let $R=0$. Since $\sum_{n}\left\|G_{0} \varepsilon_{n}\right\|_{A}^{2}=\sum_{n \in \mathcal{I}_{2}}\left\|\left(T^{\dagger} F+W_{0}\right) \varepsilon_{n}\right\|_{A}^{2}+\sum_{n \in \mathcal{I}_{1}}\left\|R_{0} \varepsilon_{n}\right\|_{A}^{2}$, it follows that for every $W \in L\left(N(F)^{\perp}, \mathcal{S}^{\perp}\right)$,

$$
\sum_{n \in \mathcal{I}_{2}}\left\|T^{\dagger} F \varepsilon_{n}+W_{0} \varepsilon_{n}\right\|_{A}^{2} \leq \sum_{n \in \mathcal{I}_{2}}\left\|T^{\dagger} F \varepsilon_{n}+W \varepsilon_{n}\right\|_{A}^{2}
$$

Given $y \in \mathcal{S}(y \neq 0)$, let $v=F^{*} y$. Then $v$ satisfies $T^{\dagger} F v=y$. Given $n_{0} \in$ $\mathcal{I}_{2}$, let $\varepsilon_{n_{0}}=v /\|v\|$ and $\left\{\varepsilon_{n}\right\}_{n \neq n_{0}}$ be such that $\left\{\varepsilon_{n}\right\}_{n \in \mathcal{I}_{2}}$ is an orthonormal basis of $N(F)^{\perp}$. For $x \in \mathcal{S}^{\perp}$, let $W_{x} \in L\left(N(F)^{\perp}, \mathcal{S}^{\perp}\right)$ be such that $W_{x} \varepsilon_{n_{0}}=$ $-x$ and $W_{x} \varepsilon_{n}=W_{0} \varepsilon_{n}$ for every $n \in \mathcal{I}_{2}, n \neq n_{0}$. By (5.4) it follows that

$$
\left\|\frac{y}{\|v\|}-\left(-W_{0} \varepsilon_{n_{0}}\right)\right\|_{A} \leq\left\|\frac{y}{\|v\|}-x\right\|_{A}
$$

for any $x \in \mathcal{S}^{\perp}$. Thus, $x_{0}=-\|v\| W_{0} \varepsilon_{n_{0}}$ is an $A$-WLSA of $y$. Therefore, by Corollary 4.3 , the pair $\left(A, \mathcal{S}^{\perp}\right)$ is compatible.

The following result gives an expression for a subset of $A$-curve fitting processes; notice that this expression does not depend on the frame $\mathcal{V}$, but only on the subspace $\mathcal{S}$.

Corollary 5.6. Let $A \in L(\mathcal{H})^{+}$be a trace class operator and $\mathcal{S}$ be a closed subspace of $\mathcal{H}$ such that the pair $\left(A, \mathcal{S}^{\perp}\right)$ is compatible. Then $W \in$ $\mathcal{P}_{*}(A, \mathcal{S})$ is an $A$-curve fitting process on $\mathcal{S}$.

Proof. Suppose that $\mathcal{V}=\left\{v_{n}\right\}_{n \in \mathcal{I}}$ is a frame for $\mathcal{S},\left(F, \mathcal{B}=\left\{e_{n}\right\}_{n \in \mathcal{I}}, \mathcal{K}\right)$ is the synthesis operator of $\mathcal{V}$, and $T=F F^{*}$. Let $Q \in \mathcal{P}\left(A, \mathcal{S}^{\perp}\right)$. By Theorem 5.5, if $G_{0}=(I-Q) T^{\dagger} F$ then $F G_{0}^{*}$ is an $A$-curve fitting process on $\mathcal{S}$. Since $F G_{0}^{*}=F\left((I-Q) T^{\dagger} F\right)^{*}=T T^{\dagger}\left(I-Q^{*}\right)=\left(I-Q^{*}\right)$, it follows that if $W \in \mathcal{P}_{*}(A, \mathcal{S})$, then $W$ is an $A$-curve fitting process on $\mathcal{S}$.

The previous corollary relates the set of $A$-curve fitting processes to the set of $A$-approximation processes. In fact, an $A$-curve fitting process exists if and only if an $A$-approximation process does. A similar result can be found in [17, Corollary of Theorem 5] for finite-dimensional spaces.

Acknowledgements. The authors are grateful to the referee for several useful comments and corrections.

This research was partially supported by CONICET (PIP 5272), UBACYT I023. 


\section{References}

[1] W. N. Anderson Jr. and G. E. Trapp, Shorted operators II, SIAM J. Appl. Math. 28 (1975), 60-71.

[2] T. Ando, Generalized Schur complements, Linear Algebra Appl. 27 (1979), 173-186.

[3] E. Andruchow, G. Corach and D. Stojanoff, Geometry of oblique projections, Studia Math. 137 (1999), 61-79.

[4] J. Antezana and G. Corach, Sampling theory, oblique projections and a question by Smale and Zhou, Appl. Comput. Harmon. Anal. 21 (2006), 245-253.

[5] J. Antezana, G. Corach, M. Ruiz and D. Stojanoff, Nullspaces and frames, J. Math. Anal. Appl. 309 (2005), 709-723.

[6] C. de Boor, Convergence of abstract splines, J. Approx. Theory 31 (1981), 80-89.

[7] G. Corach and A. Maestripieri, Weighted generalized inverses, oblique projections and least squares problems, Numer. Funct. Anal. Optim. 26 (2005), 659-673.

[8] G. Corach, A. Maestripieri and D. Stojanoff, Oblique projections and Schur complements, Acta Sci. Math. (Szeged) 67 (2001), 337-256.

[9] - - - - Generalized Schur complements and oblique projections, Linear Algebra Appl. 341 (2002), 259-272.

[10] - - - - Oblique projections and abstract splines. J. Approx. Theory 117 (2002), 189-206.

[11] F. Deutsch, The angle between subspaces in Hilbert spaces, in: Aproximation Theory, Wavelets and Applications, S. P. Singh (ed.), Kluwer, 1995, 107-130.

[12] Y. Eldar, Sampling with arbitrary samling and reconstruction spaces and oblique dual frame vectors, J. Fourier Anal. Appl. 9 (2003), 77-96.

[13] Y. Eldar and T. Werther, General framework for consistent sampling in Hilbert spaces, Int. J. Wavelets Multiresolut. Inf. Process. 3 (2005), 497-509.

[14] J. Giribet, A. Maestripieri and F. Martínez Pería, Shorting selfadjoint operators in Hilbert spaces, Linear Algebra Appl. 428 (2008), 1899-1911.

[15] S. Izumino, Convergence of generalized inverses and spline projectors, J. Approx. Theory 38 (1983), 269-278.

[16] P. Massey and D. Stojanoff, Generalized Schur complements and P-complementable operators, Linear Algebra Appl. 393 (2004), 299-318.

[17] A. Sard, Approximation and variance, Trans. Amer. Math. Soc. 73 (1952), 428-446.

[18] S. Smale and D. X. Zhou, Shannon sampling and function reconstruction from point values, Bull. Amer. Math. Soc. 41 (2004), 279-305.

Instituto Argentino de Matemática - CONICET

and Departamento de Matemática, FI-UBA

Saavedra 15 , piso 3

CP1083, Buenos Aires, Argentina

E-mail: gcorach@fi.uba.ar

jgiribet@fi.uba.ar

amaestri@fi.uba.ar

Received September 15, 2008

Revised version February 11, 2009

(6407) 